\title{
Freqüência de precordialgia em mulheres chagásicas e não-chagásicas
}

\author{
Chest pain frequency in chagasic and non-chagasic women
}

\author{
Vitorino Modesto dos Santos, Selma Freire de Carvalho da Cunha, Jenner \\ Arruda Modesto dos Santos, Taciana Arruda Modesto dos Santos, \\ Lister Arruda Modesto dos Santos e Daniel Ferreira da Cunha
}

\begin{abstract}
Resumo O objetivo do estudo foi comparar a freqüência de precordialgia em mulheres chagásicas com grupo de não-chagásicas. Realizou-se estudo retrospectivo, amostral, do tipo corte transversal, com mulheres ( $n=647)$, de idade $\geq 40$ anos, chagásicas $(n=362)$ e controles $(n=285)$. Precordialgia foi definida por queixa de dor retroesternal relacionada ou não a esforço físico. As chagásicas foram classificadas nas formas indeterminada $(n=125)$, megas $(n=58)$ e cardíaca $(n$ $=179)$. A idade (57,0 $\pm 11,3$ vs $57,3 \pm 10,4$ anos) e porcentagem de brancas $(75,8 \%$ vs $77,1 \%)$ foram similares entre chagásicas e controles, respectivamente. Precordialgia foi mais freqüente ( $p$ $<0,01)$ entre chagásicas $(14,6 \%)$ que entre controles $(5,6 \%)$, com maior prevalência na forma cardíaca (risco relativo $=2,41$; variação: 1,38-4,23), fenômeno possivelmente relacionado com distúrbios de inervação autonômica cardíaca ou esofágica, ou da inflamação em território da microcirculação coronariana.
\end{abstract}

Palavras-chaves: Doença de Chagas. Precordialgia. Miocardiopatia.

Abstract The aim of this study was to compare the frequency of precordialgia between chagasic and non-chagasic women. A cross-sectional study comprised 647 female aged $\geq 40$, chagasic $(n=$ 362) and controls $(n=285)$ was done at a Brazilian university hospital. Chagasic were classified as cardiac $(n=179)$, megas $(n=58)$ or indeterminate $(n=125)$ clinical forms. Chest pain was ascertained by typical or atypical retrosternal pain. Age (57.0 \pm 11.3 vs $57.3 \pm 10.4$ years), and percentage of white women $(75.8 \%$ vs $77.1 \%)$ were similar between chagasic and controls, respectively. Chest pain was more prevalent $(p<0.01)$ among chagasic $(14.6 \%)$ than controls $(5.6 \%)$, mainly in the cardiac form (relative risk $=2.41$; range: $1.38-4.23$ ), a phenomenon possibly related to cardiac parasympathetic denervation and myocardial microvascular changes.

Key-words: Chagas'disease. Precordial pain. Cardiomyopathy

Departamento de Clínica Médica e Curso de Pós-graduação em Patologia Humana da Faculdade de Medicina do Triângulo Mineiro, Uberaba, MG.

Endereço para correspondência: Prof. Daniel Ferreira da Cunha. Disc. de Nutrologia/Depto de Clínica Médica/HE/FMTM. Av. Getúlio Guaritá s/no 0 , 38040-040 Uberaba,MG.

Telefone: (034) 318-5335, FAX: 312-6640. E-mail: dfnutro@mednet.com.br.

Recebido para publicação em 23/06/97. 
Dor precordial atípica é uma queixa comum na prática clínica cotidiana, acarretando ansiedade, sofrimento, afastamento temporário do trabalho e sobrecarga nos serviços de Saúde33. Precordialgias relacionadas ao esforço físico ou atípicas ${ }^{14}$, podem ocorrer na fase crônica da doença de Chagas, geralmente atribuídas a lesões esofágicas 26 ou cardíacas 421.

Estudos em chagásicos necropsiados, entretanto, revelam artérias coronárias normais ou mesmo de calibre aumentado, fenômeno atribuído, por alguns autores, à desnervação autonômica8 1113161719 20. Devido a estes fatos, atribuiu-se menor importância à isquemia miocárdica na gênese da forma cardíaca da doença de Chagas8 20.

A maioria dos autores considera a precordialgia como sintoma comum entre pacientes chagásicos ${ }^{14}$, embora aparentemente não haja estudos descrevendo a prevalência concomitante de dor precordial entre controles não-chagásicos. A maior parte dos estudos descrevem aspectos anatômicos das artérias coronárias e alterações inflamatórias locais 36 . Em anos recentes, entretanto, tem aumentado o número das descrições de alterações microvasculares anatômicas e funcionais incluindo, por exemplo, o espessamento da membrana basal capilar e a maior agregação plaquetária, além de trombose, microaneurismas e espasmos em vasos coronarianos distais, associados ou não a distúrbios de inervação autonômica da fase crônica da doença de Chagas6 7182728293032 .

A hipótese do presente estudo é que alterações funcionais e anatômicas na microcirculação de indivíduos chagásicos teriam repercussão clínica, verificável, por exemplo, pela maior prevalência de queixas de dor precordial. O objetivo do estudo foi comparar a freqüência de precordialgia entre mulheres com idade $\geq 40$ anos, chagásicas (em suas diversas formas clínicas) e não-chagásicas, que procuraram assistência médica num Hospital Universitário da região do Triângulo Mineiro.

\section{MATERIAL E MÉTODOS}

O estudo, retrospectivo, foi realizado no Departamento de Clínica Médica da Faculdade de Medicina do Triângulo Mineiro (FMTM), em Uberaba, MG, no período entre junho de 1993 e julho de 1995. Os nomes e registros das pacientes foram obtidos por meio de um arquivo eletrônico extraído do Computador Central do Departamento de Sistema de Informática e Métodos e do Instituto de Desenvolvimento Tecnológico do Hospital Escola da FMTM. Os dados obtidos dos prontuários do Serviço de Arquivo Médico e Estatística (SAME) do HEFMTM foram organizados em banco de dados eletrônico planejado de forma a incluir três exames sorológicos para doença de Chagas (imunofluorescência, hemaglutinação e fixação do complemento) e informações gerais sobre as pacientes, contendo o nome, o registro no SAME, a idade, a cor e a procedência. O tamanho da amostra foi calculado utilizando-se uma prevalência média esperada de precordialgia de $15 \% 14$, com faixa de variação entre 12 e $18 \%$ e nível de confiança de $99 \%$.

Utilizou-se o processo de amostragem casual e, para assegurar-se a aleatoriedade na escolha da amostra, os números dos registros $(n=3.854)$ foram dispostos em ordem crescente. Dos primeiros 800 prontuários de pacientes do sexo feminino, foram selecionados todos os casos com idade igual ou superior a 40 anos, devido à maior probabilidade de precordialgia neste grupo etário21. Optou-se pelo estudo de mulheres devido ao fato de a dor precordial de origem coronariana ser menos freqüente no sexo feminino, o que diminui a possibilidade de superposição com a dor de origem isquêmica.

Os procedimentos de coleta e registro de informações foram realizados por um único observador, visando padronização e homogeneidade nos critérios diagnósticos. As informações clínicas foram obtidas em prontuários médicos arquivados no SAME, contendo registros de dados referentes à presença ou não de precordialgia, de palpitação, de hipertensão arterial sistêmica (HAS) e de insuficiência cardíaca congestiva (ICC). As pacientes foram classificadas como brancas e não-brancas, de acordo com a cor registrada no prontuário médico, sendo consideradas nãobrancas as descritas como negras, pardas ou mulatas.

Foram designadas chagásicas as pacientes com positividade nas três reações sorológicas para a doença de Chagas e como não-chagásicas as que apresentaram as três sorologias negativas. Com base nos dados do prontuário médico, o grupo de mulheres chagásicas foi subdividido nas seguintes formas clínicas 23 26: 1). megas, englobando pacientes com megaesôfago diagnosticado à radioscopia ou à radiografia 
contrastada do esôfago; ou megacólon, mediante comprovação de ectasia do orgão no enema opaco ou pela presença de constipação intestinal crônica superior a 10 dias; 2). cardíaca, definida por alterações eletrocardiográficas sugestivas de miocardiopatia chagásica, como extrassístoles ventriculares, bloqueios atrioventriculares de $2^{\circ}$ e $3^{\circ}$ graus, bloqueio de grau avançado do ramo direito ou bloqueio divisional ântero-superior esquerdo do feixe de His ou fibrilação atrial25 ou evidência de cardiomegalia em radiografias de tórax em póstero-anterior e perfil. A dor precordial não foi considerada como critério diagnóstico da forma cardíaca da doença de Chagas; 3). embora a rotina hospitalar não incluísse estudos radiológicos do tubo digestivo e eletrocardiográficos, consideramos na forma indeterminada os casos de positividade das três reações sorológicas para doença de Chagas, na ausência de sintomas, sinais e alterações radiológicas ou eletrocardiográficas atribuíveis às outras formas clínicas da doença22 .

Precordialgia foi definida pelo relato de dor aguda ou crônica na região retroesternal, com ou sem irradiação e relacionada ou não a esforços físicos. Foram consideradas hipertensas as mulheres com história de HAS ou apresentando níveis tensionais em repouso superiores a $150 / 90 \mathrm{mmHg}$. ICC foi definida por cardiomegalia verificada ao exame físico e/ou radiológico e sinais e sintomas de congestão pulmonar ou sistêmica. Palpitação foi considerada presente quando havia relato de "batedeira" ou percepção desagradável do próprio batimento cardíaco.

As variáveis numéricas contínuas com distribuição normal foram expressas na forma de média \pm desvio padrão. O teste "t" nãopareado de Student foi empregado na comparação entre chagásicas e não-chagásicas e a análise de variância (ANOVA) foi empregada na comparação entre o grupo controle e os subgrupos de chagásicas. As proporções foram comparadas pelo teste do $\chi^{2}$ ou pelo teste exato

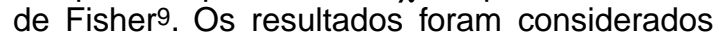
significativos quando $p<0,01$.

\section{RESULTADOS}

O levantamento de dados abrangeu 647 pacientes procedentes da região do Triângulo Mineiro, constituindo os grupos chagásicas $(n=362)$ e não-chagásicas $(n=285)$. Houve predomínio de pacientes de cor branca $(76,8 \%)$ em todos os grupos estudados, não havendo diferença estatística entre a proporção de pacientes de cor branca nas três formas clínicas e quando comparadas ao grupo controle (Tabelas 1 e 2). O grupo de pacientes da forma megas apresentou média de idade $(60,0 \pm 10,1$ anos) estatisticamente superior às médias de idade dos demais grupos.

Foram registrados 61 casos de precordialgia $(9,4 \%)$ na amostra estudada, com freqüência estatisticamente maior (risco relativo $=2,41$; variação: 1,38-4,23) em mulheres chagásicas que em não-chagásicas (12,7\% vs 5,3\%). Verificou-se diferença significativa $(p<0,01)$ na freqüência de precordialgia na forma cardíaca da doença de Chagas $(16,8 \%)$, comparativamente à indeterminada $(8,8 \%)$, à megas $(8,6 \%)$ e ao controle $(5,3 \%)$ (Tabela 2$)$.

Comparando-se o grupo chagásicas com o controle, verificou-se que apenas em relação à freqüência de insuficiência cardíaca não havia diferença significativa entre os dois grupos $(3,6 \%$ vs $4,2 \%)$. Todos os outros sintomas tiveram diferença significativa, com maior freqüência

Tabela 1 - Características da amostra de 647 mulheres com idade $\geq 40$ anos, chagásicas e controles, atendidas no HE-FMTM entre junho de 1993 e julho de 1995.

\begin{tabular}{lcc}
\hline Parâmetros & Controles $(\mathrm{n}=285)$ & Chagásicas $(\mathrm{n}=362)$ \\
\hline Idade (anos) & $57,0 \pm 11,3$ & $57,3 \pm 10,4$ \\
Cor (branca, em \%) & 75,8 & 77,1 \\
Dispnéia, $\mathrm{n}=68(\%)^{*}$ & 5,6 & 14,4 \\
Precordialgia, $\mathrm{n}=61(\%)^{*}$ & 5,3 & 12,7 \\
Palpitação, $\mathrm{n}=78(\%)^{*}$ & 6,0 & 16,8 \\
HAS, $\mathrm{n}=248(\%)$ & 38,9 & 37,8 \\
ICC, $\mathrm{n}=25(\%)$ & 4,2 & 3,6 \\
\hline
\end{tabular}

HAS = hipertensão arterial sistêmica; ICC = insuficiência cardíaca congestiva.

* Teste do $\chi^{2}, p<0,01$. 
Tabela 2 - Características da amostra de 647 mulheres com idade $\geq 40$ anos, chagásicas e controles, atendidas no HE-FMTM entre junho de 1993 e julho de 1995.

\begin{tabular}{lcccc}
\hline Parâmetros & $\begin{array}{c}\text { Controle } \\
(\mathrm{n}=285)\end{array}$ & $\begin{array}{c}\text { Indeterminada } \\
(\mathrm{n}=125)\end{array}$ & $\begin{array}{c}\text { Megas } \\
(\mathrm{n}=58)\end{array}$ & $\begin{array}{c}\text { Cardíaca } \\
(\mathrm{n}=179)\end{array}$ \\
\hline Idade (anos) & $57,0 \pm 11,3$ & $55,4 \pm 10,5$ & $60,0 \pm 10,1(\mathrm{a})$ & $57,8 \pm 10,5$ \\
Cor (branca, em \%) & 75,8 & 80,0 & 75,9 & 75,4 \\
HAS (\%) & $38,9(\mathrm{~b})$ & 26,4 & 25,9 & $49,7(\mathrm{~b})$ \\
ICC (\%) & 4,2 & 0,0 & 5,2 & 5,6 \\
Precordialgia (\%) & 5,3 & 8,8 & 8,6 & $16,8(\mathrm{c})$ \\
Dispnéia (\%) & 5,6 & $12,8(\mathrm{~d})$ & 5,2 & $18,4(\mathrm{~d})$ \\
Palpitação (\%) & $6,0(\mathrm{e})$ & 12,0 & 19,0 & 19,5 \\
\hline
\end{tabular}

HAS = hipertensão arterial sistêmica; ICC = insuficiência cardíaca congestiva. Teste do $(2, p<0,01$.

(a): Megas são estatisticamente mais velhas; (b): HAS foi mais comum no controle e forma cardíaca; (c): Precordialgia foi mais comum na forma cardíaca; (d); Dispnéia foi mais comum nas formas indeterminada e cardíaca; (e): Palpitação foi estatisticamente menos freqüente nos controles.

nas chagásicas: dispnéia $(14,4 \%$ vs $5,6 \%)$ e palpitação $(16,8 \%$ vs $6,0 \%)$. A freqüência de palpitação no grupo controle diferiu das formas cardíaca $(19,6 \%)$, megas $(19,0 \%)$ e indeterminada $(12,0 \%)$ (Tabela 2$)$.

A prevalência de HAS foi alta no material estudado ( $n=248$ ou $38,3 \%$ ). Numa primeira análise, verificou-se similaridade na freqüência de HAS entre chagásicas $(37,8 \%)$ e nãochagásicas $(38,9 \%)$. HAS foi muito mais comum na forma cardíaca $(49,7 \%)$ da doença de Chagas que na forma indeterminada $(26,4 \%)$ ou megas $(25,9 \%)$ (Tabela 2$)$.

\section{DISCUSSÃO}

No presente estudo documentou-se que as chagásicas apresentam maior prevalência de precordialgia que as não-chagásicas, pareadas para cor e idade. A maior porcentagem de casos foi observada na forma cardíaca da doença $(16,8 \%)$, o que sugere que a dor origina-se no próprio coração e não em outro órgão. Entre as explicações aventadas 21 para a maior freqüência de dor precordial nas chagásicas, pode-se incluir o espasmo do cárdia ou a esofagite em casos de megaesôfago26 31, a ocorrência de miocardite e/ou epicardite 16 , as anormalidades vasomotoras coronarianas 34 e a isquemia miocárdica relativa, decorrente da hipertrofia miocárdica, agravada ou não pela HAS21. É digno de nota que as chagásicas com a forma megas, mais idosas e presumivelmente mais propensas à cardiopatia isquêmica, não apresentaram maior freqüência de precordialgia que os controles ou as chagásicas com a forma indeterminada; este achado reforça a idéia de que a coronariopatia obstrutiva não seja a única responsável pela insuficiência coronariana na miocardite chagásica crônica3 718273034 .

Embora possa ocorrer morte súbita12 1524 em chagásicos previamente assintomáticos, uma miocardiopatia chagásica crônica histologicamente grave pode ser silenciosa do ponto de vista clínico1 2. Estudos invasivos, utilizando perfusão miocárdica com tálio-201 ou tecnécio-99 mostram que chagásicos crônicos com dor precordial atípica e coronárias normais muitas vezes apresentam anormalidades na microcirculação coronariana16. É possível que chagásicos com precordialgia atípica, atualmente classificados na forma indeterminada, sejam na verdade portadores de lesão isquêmica na microcirculação coronariana; se for verdade, pode-se esperar aumento dos níveis séricos de anticorpos de reação cruzada à miosina-B13 nesses casos 5 .

A maior concomitância de HAS no subgrupo de chagásicas com a forma cardíaca pode decorrer apenas do fato de ser a hipertensão arterial um fator importante na sobrecarga ventricular esquerda, estando associado ou não com hipertrofia, dilatação e descompensação cardíacas, além de alterações eletrocardiográficas. Outra possibilidade seria que a HAS provocasse hipertrofia do ventrículo esquerdo, levando a uma isquemia miocárdica relativa e maior prevalência de dor precordial21. Apesar de não terem sido incluídas nos critérios diagnósticos e na caracterização da forma cardíaca da doença de Chagas, a maior freqüência de dispnéia e de palpitação neste subgrupo também está de acordo com o esperado, já que esses sintomas podem corresponder à expressão clínica de 
mecanismos de adaptação cardiocirculatória, tanto à ICC como à HAS.

Dentre as limitações do estudo retrospectivo, considera-se a possibilidade de terem sido incluídos indivíduos com dor de origem psicogênica ou relacionada a uma cardiopatia isquêmica associada10 14. Outra possível falha seria o critério diagnóstico para dor precordial, baseado apenas em informações obtidas no prontuário médico. Entretanto, pelas características da metodologia empregada, que distribui de forma homogênea eventuais erros na obtenção de dados amostrais, pode-se considerar como válida a conclusão de que chagásicas apresentem maior prevalência de dor precordial atípica.

No presente estudo documentou-se que a dor precordial é mais freqüente em chagásicas que em não-chagásicas (risco relativo $=2,41$; variação: $1,38-4,23)$, fenômeno que pode estar relacionado com isquemia no território coronariano e/ou com distúrbios na motilidade esofágica. Os resultados deste estudo dão suporte à hipótese de que, na doença de Chagas, as dores precordiais decorram da cardiopatia que o $T$. cruzi provoca direta ou indiretamente.

Estudos prospectivos que incluíssem subgrupos de chagásicos, com ou sem HAS e com ou sem coronariopatia obstrutiva documentada por cinecoronariografia, permitiriam avaliar melhor um possível papel de distúrbios microcirculatórios na gênese de dores torácicas comumente relatadas por chagásicos crônicos.

\section{AGRADECIMENTOS}

Aos funcionários do IDT-DSIM, Mário Roberto Ferreira e Dalva Pereira da Silva, pela assessoria técnica. À Sra. Simone Amaral Coelho de Oliveira, pelo apoio no trabalho de digitação dos dados.

\section{REFERÊNCIAS BIBLIOGRÁFICAS}

1. Adad SJ, Lopes ER, Chapadeiro E. Estudo comparativo entre as lesões anátomo-patológicas no coração e esôfago de chagásicos crônicos. Revista da Sociedade Brasileira de Medicina Tropical 24:15, 1991.

2. Almeida HO. Hipóxia como fator patogenético da lesão apical em chagásicos crônicos. Revista Goiana de Medicina 32:129-139, 1986.

3. Almeida HO, Teixeira VPA, Gobbi H, Rocha A, Brandão MC. Inflamação associada a células musculares cardíacas parasitadas pelo Trypanosoma cruzi em chagásicos crônicos. Arquivos Brasileiros de Cardiologia 42:183-186, 1984.

4. Amorim DS, Marin-Neto JA. Functional alterations of the autonomic nervous system in Chagas' heart disease. São Paulo Medical Journal/Revista Paulista de Medicina, 113:772-784, 1995.

5. Cunha Neto A, Gruber A, Zingales B, Kalil J. Estudo da doença de Chagas: abordagem molecular. Revista da Sociedade de Cardiologia do Estado de São Paulo 5:217-229, 1995.

6. Factor SM, Cho S, Wittner M, Tanowitz H. Abnormalities of the coronary microcirculation in acute murine Chagas' disease. American Journal of Tropical Medicine and Hygiene 34:246-253, 1985.

7. Ferrans VJ, Milei J, Storino RA. Basement membrane thickening in cardiac myocytes and capillaries in chronic Chagas' disease. American Journal of Cardiology 61:1137-1140, 1986.

8. Ferreira CS, Lopes ER, Chapadeiro E, Almeida HO, Souza WF, Neto IJS. Coronariografia post mortem na cardite chagásica crônica. Correlação anátomoradiológica. Arquivos Brasileiros de Cardiologia 34:81-86, 1980.

9. Gantz SA. Primer of Biostatistics. 4th edition. McGraw-Hill, New York, 1997.

10. Garzon SAG. Doença de Chagas associada a outras cardiopatias. In: Cançado JR., Chuster, M (ed) Doença de Chagas. Fundação Carlos Chagas, Belo Horizonte, p.255-265, 1985.

11. Hagar JH, Rahimtoola SH. Chagas' heart disease in the United States. New England Journal of Medicine 325:763-768, 1991.

12. Junqueira-Júnior LF. Disfunção autonômica cardíaca, arritmogênese e morte súbita chagásica: perspectiva fisiopatológica. Revista da Sociedade Brasileira de Medicina Tropical 26:30-35, 1993.

13. Köberle F. Cardiopatia chagásica. Hospital 53:311346, 1958.

14. Lopes ER. Origem esofágica de dor precordial em chagásicos. Arquivos Brasileiros de Cardiologia 64:153-154, 1995.

15. Lopes ER, Chapadeiro E, Almeida HO, Rocha A. Contribuição ao estudo da anatomia patológica dos corações de chagásicos falecidos subitamente. Revista da Sociedade Brasileira de Medicina Tropical 9:269-282, 1975.

16. Marin Neto JA, Simões MV, Ayres-Neto EM, Santos JLA, Gallo Júnior L, Amorim DS, Maciel BC. Studies of the coronary circulation in Chagas' heart disease. 
São Paulo Medical Journal/Revista Paulista de Medicina 113:826-834, 1995.

17. Mesquita PM, Mesquita LFV, Meneses AAC, Costa Neto B, Marchi R, Lopes ER. Freqüência de arteriosclerose coronária e infarto do miocárdio em chagásicos crônicos. Revista da Sociedade Brasileira de Medicina Tropical 24:15-16, 1991.

18. Morris SA, Tanowitz HB, Wittner M, Bilezikian JP. Pathophysiological insights into the cardiomyopathy of Chagas' disease. Circulation 82:1900-1909, 1990.

19. Oliveira JSM. Tromboses cardíacas e tromboembolismo. In: Cançado J R, Chuster M (eds) Doença de Chagas. Fundação Carlos Chagas, Belo Horizonte, p.54-60, 1985.

20. Oliveira JSM, Santos JCM, Muccillo G, Ferreira AL. Increased capacity of the coronary arteries in chronic Chagas' heart disease:further support for the neurogenic pathogenesis concept. American Heart Journal 109:304-308, 1985.

21. O'Rourke RA. Chest pain. In: Schlant RC, Alexander RW (eds) Hurst's the Heart: arteries and veins, 8th edition. McGraw-Hill, New York, p.459-467, 1994.

22. Pereira Barreto AC, lanni BM. The indetermined form of Chagas' heart disease: concept and forensic implications. São Paulo Medicine Journal/Revista Paulista de Medicina 113:797-801, 1995.

23. Prata A. Chagas' disease. Infectious Diseases Clinics of North America 8: 61-76, 1994.

24. Prata A, Lopes ER, Chapadeiro E. Características da morte súbita tida como não esperada na doença de Chagas. Revista da Sociedade Brasileira de Medicina Tropical 19:9-12, 1986.

25. Prata SP, Cunha DF, Cunha SFC, Prata SC, Nogueira N. Prevalência de alterações eletrocardiográficas em 2000 pacientes chagásicos idosos e não-idosos. Arquivos Brasileiros de Cardiologia 60:369-372, 1993.

26. Rezende JF. Manifestações digestivas da doença de Chagas. In: Dani R, Paula Castro L (eds)
Gastroenterologia Clínica, 3a edição. Guanabara, Rio de Janeiro, p.1729-1755, 1993.

27. Rossi MA. Microvascular changes as a cause of chronic cardiomyopathy in Chagas'disease. American Heart Journal 120:233-236, 1990.

28. Rossi MA, Carobrez SG. Experimental Trypanosoma cruzi cardiomyopathy in BALB/c mice:histochemical evidence of hypoxic changes in the myocardium. British Journal of Experimental Pathology 66:155161, 1985.

29. Rossi MA, Gonçalves S, Ribeiro dos Santos R. Experimental Trypanosoma cruzi cardiomyopathy in $B A L B / c$ mice. The potential role of intravascular platelet aggregation in its genesis. American Journal of Pathology 114:209-216, 1984.

30. Rossi MA, Ramos SG. Pathogenesis of chronic Chagas' disease. Cardiovasccular Pathology 15:197202, 1996.

31. Simões MV, Dantas RO, Ejima FH, Meneguelli UG, Maciel BC, Marin-Neto JA. Origem esofágica de dor precordial em pacientes chagásicos com artérias coronárias subepicárdicas normais. Arquivos Brasileiros de Cardiologia 64:103-107, 1995.

32. Tanowitz HB, Burns ER, Sinha AK, Kahn NN, Morris SA, Factor SM, Hatcher VB, Bilezikian JP, Baum SG, Wittner M. Enhanced platelet adherence and aggregation in Chagas' disease: a potential pathogenic mechanism for cadiomyopathy. American Journal of Medicine Tropical and Hygiene 43:274281, 1990.

33. Teixeira MJ, Shibata MK, Pimenta CAM, Corrêa CF (eds) Dor no Brasil: estado atual e perspectivas. Limay, São Paulo, 1995.

34. Torres FW, Acquatella H, Condado JA, Dinsmore R, Palacios IF. Coronary vascular reactivity is abnormal in patients with Chagas' heart disease. American Heart Journal 129:995-1001, 1995. 